Revue d'histoire du XIXe siècle

Société d'histoire de la révolution de 1848 et des

révolutions du XIXe siècle

$53 \mid 2016$

Mobilités, savoir-faire et innovations

Marc LELEUX, Aux sources de la précarité. L'instrumentalisation du travail dans le Nord, 1848-1914

Villeneuve d'Ascq, Presses universitaires du Septentrion, 2015

François Jarrige

\title{
OpenEdition
}

Journals

Édition électronique

URL : http://journals.openedition.org/rh19/5156

DOI : $10.4000 /$ rh 19.5156

ISSN : $1777-5329$

Éditeur

La Société de 1848

Édition imprimée

Date de publication : 1 décembre 2016

Pagination : 213-216

ISSN : 1265-1354

Référence électronique

François Jarrige, « Marc LELEUX, Aux sources de la précarité. L'instrumentalisation du travail dans le Nord, 1848-1914 », Revue d'histoire du XIXe siècle [En ligne], 53 | 2016, mis en ligne le 21 février 2017, consulté le 23 septembre 2020. URL : http://journals.openedition.org/rh19/5156 ; DOI : https:// doi.org/10.4000/rh19.5156

Ce document a été généré automatiquement le 23 septembre 2020.

Tous droits réservés 


\section{Marc LELEUX, Aux sources de la précarité. L'instrumentalisation du travail dans le Nord, 1848-1914}

Villeneuve d'Ascq, Presses universitaires du Septentrion, 2015

\section{François Jarrige}

\section{RÉFÉRENCE}

Marc LELEUX, Aux sources de la précarité. L'instrumentalisation du travail dans le Nord, 1848-1914, Villeneuve d'Ascq, Presses universitaires du Septentrion, 2015, 273 p., $28 €$.

1 Poursuivant l'exploration de l'histoire des travailleurs du Nord et de leur précarité, déjà entamée dans un ouvrage précédent qui portait sur le $\mathrm{XX}^{\mathrm{e}}$ siècle ${ }^{1}$, Marc Leleux propose ici de revenir « aux sources de la précarité » en examinant l'entrée progressive des ouvriers dans l'âge de la grande industrie et du salariat, qu'il situe entre la grave crise du milieu du XIX ${ }^{\mathrm{e}}$ siècle et le déclenchement de la Grande Guerre. Publié lorsque le gouvernement censé être socialiste élaborait une série de projets de réformes du marché du travail d'inspiration libérale, cet ouvrage d'histoire s'inscrit résolument dans les enjeux du présent. Alors que la loi dite travail a finalement été adoptée en 2016, beaucoup d'observateurs ont analysé ce texte comme un retour au XIX siècle et à sa précarité. L'ouvrage de Marc Leleux se comprend dans ce contexte: de nombreux traits du monde actuel rappellent en effet la situation des travailleurs précaires et sans travail du XIX $\mathrm{X}^{\mathrm{e}}$ siècle. La " précarité du travail»- expression qui aurait mérité d'être davantage définie et discutée - constitue ainsi une expérience essentielle des débuts de l'âge industriel, lorsque l'ancienne proto-industrie s'effrite au profit des usines concentrées et que les anciens tisserands à domicile deviennent progressivement tisseurs en usine.

2 L'histoire de l'industrialisation du Nord, des mutations de la proto-industrie, de l'émergence d'un système usinier concentré avec ses machines, son patronat et sa 
classe ouvrière, ses souffrances au quotidien et ses luttes héroïques, est évidemment bien connue. À cet égard, et même s'il retourne aux sources, l'ouvrage de Marc Leleux s'apparente surtout à une synthèse. L'auteur, chercheur à l'université de Lille 3, poursuit une tradition locale d'histoire sociale des mondes $\mathrm{du}$ travail et de l'industrialisation. Il s'inscrit également dans une série de renouvellements et dans un renouveau d'intérêt pour l'histoire du travail, un temps en crise, que traduit par exemple la création en 2013 de l'Association française pour l'histoire des mondes du travail (AFHMT). Les références sont certes parfois un peu datées: pourquoi citer uniquement le vieux livre d'Édouard Dolléans lorsqu'il est question du chartisme britannique alors que tant de riches travaux ont été consacrés à ce mouvement, qui auraient utilement pu nourrir la réflexion de l'auteur sur les relations entre mondes du travail et politique ${ }^{2}$. L'étude est construite de façon assez classique et explore tour à tour le marché du travail et ses structures, les réactions et mobilisations ouvrières à la misère et au sous-emploi chronique, et enfin la prise en charge de la question par le champ politique. Elle démarre sur la "grande peur ouvrière » du milieu du XIX ${ }^{\mathrm{e}}$ siècle, sur la «révélation de 1848 » qui impose le chômage comme enjeu central et place les travailleurs de la grande industrie au centre des regards.

Dans le Nord, la crise économique et les bouleversements politiques de 1848 poussent en effet à l'éclosion de nombreuses revendications et révoltes, les mineurs d'Anzin exigent l'augmentation des salaires alors que d'autres dénoncent les machines ou les travailleurs belges rendus responsables de leur misère. Face à la crise, les autorités cherchent à fournir du travail par divers moyens pour ramener le calme alors que les ouvriers élaborent des ébauches d'organisations collectives sous la forme de sociétés de résistances et d'associations. Dans les décennies qui suivent, du Second Empire à la Troisième République, le département connaît d'importantes transformations économiques. Précocement industrialisé, le Nord se transforme avec le développement $\mathrm{du}$ réseau ferroviaire, l'urbanisation, les mutations techniques, l'insertion croissante dans des marchés mondiaux, autant d'éléments qui remodèlent le fonctionnement du marché du travail et entretiennent une instabilité et une précarité permanentes. Si l'industrialisation crée certes du travail, elle entretient également le sous-emploi chronique et une misère ouvrière qui frappe les contemporains qui tentent de l'atténuer via la bienfaisance privée et l'assistance publique.

Dans un second temps l'auteur décrit les « réactions et mobilisations ouvrières » à cette situation. Il distingue classiquement les "réactions spontanées» (celles qui relèveraient en quelque sorte d'une forme d'enfance du mouvement ouvrier, à l'image de l'hostilité contre les machines ou du rejet des travailleurs immigrés) et les formes d'organisation syndicales et politiques plus modernes qui commencent à voir le jour. Les développements les plus originaux sont sans doute ceux du chapitre 6 consacrés aux «essais d'organisation des sans-travail» à partir des années 1880 lorsque s'accentue le chômage. À côté des organisations syndicales s'ébauche en effet un mouvement de sans-travail parfois influencé par les militants anarchistes. L'auteur montre très bien combien l'introduction des nouvelles machines reste une préoccupation permanente dans le Nord, mais sans réellement rentrer dans le détail des processus de mécanisation et ce qu'ils modifient à l'expérience ouvrière. L'ouvrage se termine par une troisième partie plus courte consacrée à la prise en charge politique : comment agissent les différents régimes successifs à l'égard des travailleurs précaires du Nord? Pour qui votent les ouvriers? Comment réagissent-ils à l'installation du régime républicain? Autant de questions classiques qui permettent 
d'explorer comment les ouvriers investissent l'arène politique, profitent du suffrage universel et de la république pour tenter de faire reconnaitre leurs revendications et s'assurer du travail. Dans le Nord plus qu'ailleurs, le paysage politique est marqué par l'importance de la démocratie chrétienne et la forte implantation socialiste, et la question ouvrière impose le travail et le sous-emploi comme des enjeux essentiels. Selon la coloration politique, les réponses apportées afin de séduire l'électorat ouvrier sont évidemment très diverses.

5 L'ouvrage de Marc Leleux propose en définitive une vaste traversée du second XIX siècle sous l'angle du sous-emploi chronique qui caractérise le monde du travail de cette époque, ses causes, ses formes, les réactions et premières réponses qu'il suscite. Evidemment, au cours de cette période de transition c'est la nature même de ce qu'il appelle « sous-emploi » qui se transforme : d'un phénomène presque naturel, prévisible et essentiellement saisonnier dans les sociétés rurales et proto-industrielles anciennes, le sous-emploi devient plus brutal et dépendant de l'instabilité des marchés et des choix patronaux. On passe d'une pauvreté ancienne à une précarité nouvelle, peut-être plus difficile à vivre car elle s'accompagne aussi d'une déstructuration des rapports sociaux anciens. La grande industrie concentrée a généré des formes de sous-emploi inédites qui menaçaient les fondements même du capitalisme, d'où l'intervention croissante de l'État et l'adoption de mesures sociales, non pour freiner l'essor du capitalisme industriel mais plutôt pour que celui-ci «puisse poursuivre son évolution sans être perturbé par un risque révolutionnaire » (p. 250). L'exemple du Nord - un des départements les plus ouvriers et industriels du pays - sert ici de terrain pour comprendre comment s'est construit le compromis historique qui a fondé l'expansion des sociétés industrialisées au cours du $\mathrm{XX}^{\mathrm{e}}$ siècle, et qui tend à entrer en crise aujourd'hui. C'est pourquoi il aurait sans doute été utile de davantage mettre en regard la situation du Nord avec d'autres configurations en France et ailleurs. Au XX ${ }^{\mathrm{e}}$ siècle, l'importance du monde ouvrier, sa centralité sociale et politique dans la continuité de la période d'industrialisation antérieure, a maintenu une forme d'équilibre et rendu possible la conquête de nombreux droits sociaux et de nombreuses sécurités. Ceux-ci ont indéniablement permis d'atténuer l'instabilité du marché du travail, la misère et le sous-emploi chronique qui existaient avant 1914. Mais il aurait sans doute été utile de rappeler combien ce compromis, toujours fragile, qui caractérise les sociétés industrialisées du Nord s'est aussi construit sur une inégalité globale à l'échelle du monde, sur l'exploitation intensive des hommes et des milieux naturels dans d'autres territoires. C'est ce compromis qui ne cesse de s'effriter depuis les années 1980. La montée en puissance de la précarité, dans la réalité du marché du travail comme dans les discours, témoigne de l'ouverture d'une nouvelle phase incertaine du capitalisme industriel qui ne sera évidemment jamais un simple retour au XIX ${ }^{\mathrm{e}}$ siècle, même s'il peut en rappeler certains traits. 


\section{NOTES}

1. Marc Leleux, Histoire des sans-travail et des précaires du Nord, Villeneuve d'Ascq, Presses universitaires du Septentrion, 2013.

2. Nous pensons évidemment aux travaux de Gareth Stedman-Jones et aux débats qui les ont suivis, ou à la riche synthèse récemment traduite en français de Malcom Chase, Chartism : A New History, Manchester, Manchester University Press, 2007, trad. française : Le Chartisme : Aux origines du mouvement ouvrier britannique (1838-1858), Paris, Publications de la Sorbonne, 2013. 